\title{
The coincidence Nielsen number for maps into real projective spaces
}

\author{
by \\ Jerzy Jezierski (Warszawa)
}

\begin{abstract}
We give an algorithm to compute the coincidence Nielsen number $N(f, g)$, introduced in $[\mathrm{DJ}]$, for pairs of maps into real projective spaces.

1. Preliminaries. Let $f, g: M \rightarrow N$ be a pair of maps between closed $C^{1}$-smooth connected manifolds of the same dimension. We investigate the coincidence set $\Phi(f, g)=\{x \in M: f x=g x\}$ of such a pair. The Nielsen relation $(x, y \in \Phi(f, g)$ are Nielsen equivalent iff there is a path $\omega$ from $x$ to $y$ such that $f \omega$ and $g \omega$ are fixed-end-homotopic) divides $\Phi(f, g)$ into Nielsen classes ([J], [M]). We will denote the quotient set by $\Phi^{\prime}(f, g)$. If $M$ and $N$ are orientable then we use the classical coincidence index [V] to define essential and nonessential classes and the Nielsen number [M]. If the orientability assumption is dropped we use the coincidence semi-index introduced in [DJ].

We recall briefly its definition. We consider a transverse pair of maps $f, g: M \rightarrow N$, i.e. for any $x \in \Phi(f, g)$ the graphs $\Gamma_{f}, \Gamma_{g} \subset M \times N$ are transverse at the point $(x, f x=g x)$. Let $x, y \in \Phi(f, g)$ and let the path $\omega$ establish the Nielsen relation between them. Fix local orientations $\alpha_{0}(f)$, $\alpha_{0}(g)$ of the graphs $\Gamma_{f}, \Gamma_{g}$ at the point $(x, f x=g x)$. Let $\alpha_{t}(f), \alpha_{t}(g)$ denote their translations along the paths $(\omega, f \omega),(\omega, g \omega)$ in $\Gamma_{f}$ and $\Gamma_{g}$ respectively. Then their sum $\alpha_{0}=\alpha_{0}(f) \wedge \alpha_{0}(g)$ is an orientation of $M \times N$ at $(x, f x)$; let $\alpha_{t}$ be its translation along $(\omega, f \omega)$ in $M \times N$. We say that $x$ and $y$ are $R$-related (reduce each other in [DJ]) iff $\alpha_{1}=-\alpha_{1}(f) \wedge \alpha_{1}(g)$ for a path $\omega$ establishing the Nielsen relation.
\end{abstract}

Now we may represent a Nielsen class $A$ as

$$
A=\left\{a_{1}, b_{1}, \ldots, a_{k}, b_{k}: c_{1}, \ldots, c_{s}\right\}
$$

where $a_{i} R b_{i}$ but no pair $\left\{c_{i}, c_{j}\right\}$ satisfies this relation $(i \neq j)$. Finally, we define the semi-index of this class:

$$
|\operatorname{ind}|(f, g: A):=s .
$$


It turns out that this definition applied in the orientable case gives the absolute value of the ordinary coincidence index. Now we follow the standard method of generalizing the Nielsen theory to the non-orientable case.

We will also use the coincidence Lefschetz number $L(f, g)$ defined in [V] for pairs of maps $f, g: M \rightarrow N$ between closed orientable $n$-manifolds. This definition may be repeated for $\mathbb{Z}_{2}$ coefficients with no orientability assumptions: for $k=0, \ldots, n$ we consider the squares

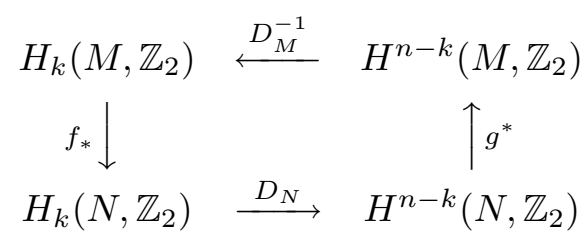

where $D_{M}, D_{N}$ stand for the Poincaré duality. We set $\Theta_{k}=D_{M}^{-1} g^{*} D_{N} f_{*}$ : $H_{k}\left(M, \mathbb{Z}_{2}\right) \rightarrow H_{k}\left(M, \mathbb{Z}_{2}\right)$ and define the coincidence Lefschetz number $\bmod 2: L_{2}(f, g)=\sum_{k=0}^{n} \operatorname{tr} \Theta_{k} \in \mathbb{Z}_{2}$. Then

$$
L_{2}(f, g)= \begin{cases}0 & \text { if }|\operatorname{ind}|(f, g) \text { is even } \\ 1 & \text { if }|\operatorname{ind}|(f, g) \text { is odd }\end{cases}
$$

so if $M$ and $N$ are oriented then $L_{2}(f, g)=\varrho L(f, g)$ where $\varrho: \mathbb{Z} \rightarrow \mathbb{Z}_{2}$ is the epimorphism.

Let $A$ be a Nielsen class of a transverse pair $f, g$. We call this class defective iff $x R x$ for some $x \in A$ [Je2, Section 2]. Then any two points in $A$ are $R$-related [Je2, (2.3)] and therefore

$$
\mid \text { ind } \mid(f, g: A)= \begin{cases}0 & \text { if } \# A \text { is even } \\ 1 & \text { if } \# A \text { is odd }\end{cases}
$$

(\# denotes cardinality). In particular, if $\Phi(f, g)$ is a unique Nielsen class and is defective then by abuse of notation $N(f, g)=L_{2}(f, g)$.

We will also need the following two lemmas.

(1.1) Lemma. Let $p: \widetilde{M} \rightarrow M$ be a covering, $M, \widetilde{M}$ manifolds, $\widetilde{M}$ oriented. Let $\alpha: \widetilde{M} \rightarrow \widetilde{M}$ be the covering transformation corresponding to $a \in \pi_{1}\left(M, x_{0}\right)$. Then the map $\alpha$ is orientation-preserving on $\widetilde{M}$ iff $a$ preserves local orientation at $x_{0} \in M$.

(1.2) Lemma. Let $f: M \rightarrow \mathbb{R} P^{n}$ be a map from an $n$-dimensional manifold $M$ into the real projective space $\mathbb{R} P^{n}$ ( $n$ even) such that there exists $a \in \pi_{1} M$ preserving local orientation of $M$ and $f_{\#} a \neq 0$. Let $p: \widetilde{M} \rightarrow M$ be a finite covering such that $\widetilde{M}$ is orientable and $a \notin p_{\#} \pi_{1} \widetilde{M}$. Then any 
lift $\widetilde{f}$,

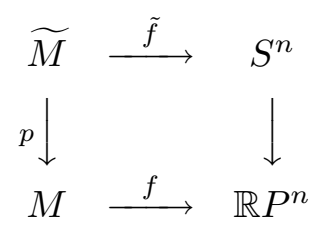

is freely homotopic to a constant map.

Proof. Let $\alpha: \widetilde{M} \rightarrow \widetilde{M}$ be the covering transformation corresponding to $a \in \pi_{1} M$ and let $\beta: S^{n} \rightarrow S^{n}$ denote the antipodism. Then the diagram

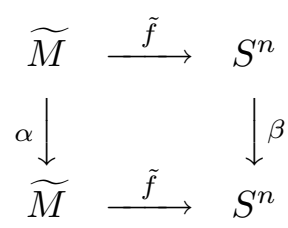

is commutative and hence $\operatorname{deg}(\widetilde{f} \alpha)=\operatorname{deg}(\beta \widetilde{f})$. But by (1.1)

$$
\begin{aligned}
& \operatorname{deg}(\tilde{f} \alpha)=\operatorname{deg} \widetilde{f} \operatorname{deg} \alpha=+\operatorname{deg} \widetilde{f}, \\
& \operatorname{deg}(\beta \widetilde{f})=\operatorname{deg} \beta \operatorname{deg} \tilde{f}=-\operatorname{deg} \widetilde{f},
\end{aligned}
$$

hence $\operatorname{deg} \tilde{f}=0$ and the Hopf theorem implies our lemma.

2. Covering spaces. Let $M, N$ be closed connected smooth manifolds of the same dimension and let $p: \widetilde{M} \rightarrow M, q: \widetilde{N} \rightarrow N$ be finite connected regular coverings coresponding to the normal subgroups $H=\operatorname{im} p_{\#} \subset \pi_{1} M$, $H^{\prime}=\operatorname{im} q_{\#} \subset \pi_{1} N$. Let $\Gamma_{M}, \Gamma_{N}$ denote the groups of covering transformations of these coverings. Let $f, g: M \rightarrow N$ be a pair of maps admitting lifts:

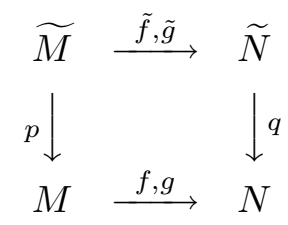

In this section we find a formula expressing the Nielsen number $N(f, g)$ by the numbers $N(\widetilde{f}, \widetilde{g})$. First we notice that $\Phi(f, g)=\bigcup p \Phi(\widetilde{f}, \widetilde{g})$ where the summation runs over all pairs of lifts. The sets $p \Phi(\widetilde{f}, \widetilde{g})$ are either disjoint or equal and each of them is a sum of some Nielsen classes of $f, g$. Fix $x_{0} \in \Phi(f, g)$ and let $f_{\#}^{H}, g_{\#}^{H}: \pi_{1}\left(M, x_{0}\right) / H\left(x_{0}\right) \rightarrow \pi_{1}\left(N, f x_{0}\right) / H^{\prime}\left(f x_{0}\right)$ 
denote the homomorphisms induced by $f$ and $g$ respectively. Let

$$
\begin{aligned}
C\left(f_{\#}, g_{\#}\right)_{x_{0}} & =\left\{\langle a\rangle \in \pi_{1}\left(M, x_{0}\right): f_{\#}\langle a\rangle=g_{\#}\langle a\rangle\right\}, \\
C\left(f_{\#}^{H}, g_{\#}^{H}\right)_{x_{0}} & =\left\{\langle a\rangle_{H} \in \pi_{1}\left(M, x_{0}\right) / H\left(x_{0}\right): f_{\#}^{H}\langle a\rangle_{H}=g_{\#}^{H}\langle a\rangle_{H}\right\}, \\
\bar{C}\left(f_{\#}, g_{\#}\right)_{x_{0}} & =\operatorname{im}\left\{j^{H}: C\left(f_{\#}, g_{\#}\right)_{x_{0}} \rightarrow C\left(f_{\#}^{H}, g_{\#}^{H}\right)_{x_{0}}\right\}
\end{aligned}
$$

where $j^{H}$ denotes the natural projection of $\pi_{1} M$ onto $\pi_{1} M / H$. Finally, let $P: C\left(f_{\#}^{H}, g_{\#}^{H}\right)_{x_{0}} \rightarrow C\left(f_{\#}^{H}, g_{\#}^{H}\right)_{x_{0}} / \bar{C}\left(f_{\#}, g_{\#}\right)_{x_{0}}$ (left cosets) be the natural projection.

(2.1) Lemma. Fix a pair of lifts $\widetilde{f}, \widetilde{g}$ and points $x_{0} \in p \Phi(\widetilde{f}, \widetilde{g}), \widetilde{x}_{0} \in$ $p^{-1}\left(x_{0}\right) \cap \Phi(\widetilde{f}, \widetilde{g})$. For any $\widetilde{x} \in p^{-1} x_{0} \cap \Phi(\widetilde{f}, \widetilde{g})$ fix a path $\widetilde{\omega}$ from $\widetilde{x}_{0}$ to $\widetilde{x}$. Define the map $\Theta: p^{-1} x_{0} \cap \Phi(\widetilde{f}, \widetilde{g}) \rightarrow C\left(f_{\#}^{H}, g_{\#}^{H}\right)_{x_{0}}$ putting $\Theta(\widetilde{x})=\langle p \widetilde{\omega}\rangle_{H}$. Then

(a) $\Theta$ is a well defined bijective map.

(b) (naturality) Let $x_{1}$ be another coincidence point of $\Phi(f, g)$ lying in the same Nielsen class. Let $\tau$ be a path establishing the Nielsen relation between $x_{0}$ and $x_{1}$. Then the diagram

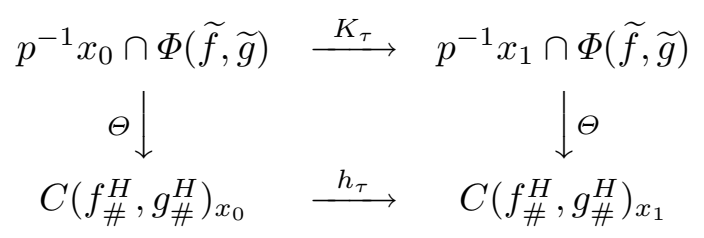

is commutative $\left(K_{\tau}(\widetilde{x})=\widetilde{\tau}(1)\right.$ where $\widetilde{\tau}$ is the lift of $\tau$ starting at $\widetilde{x}$ and $h_{\tau}$ is given by $\left.h_{\tau}\langle\omega\rangle_{H}=\langle-\tau+\omega+\tau\rangle_{H}\right), K_{\tau}$ is a bijection and $h_{\tau}$ is a group isomorphism.

(c) $\widetilde{x}_{1}, \widetilde{x}_{2} \in p^{-1} x_{0} \cap \Phi(\widetilde{f}, \widetilde{g})$ are Nielsen equivalent iff $P \Theta\left(\widetilde{x}_{1}\right)=P \Theta\left(\widetilde{x}_{2}\right)$ $\in C\left(f_{\#}^{H}, g_{\#}^{H}\right)_{x_{0}} / \bar{C}\left(f_{\#}, g_{\#}\right)_{x_{0}}$.

Pr o of. (a) and (b) follow from easy calculations. We prove (c). Assume that $\widetilde{x}_{1}$ and $\widetilde{x}_{2}$ are Nielsen equivalent. Fix paths $\widetilde{\omega}$ from $\widetilde{x}_{1}$ to $\widetilde{x}_{2}$ satisfying $\widetilde{f} \widetilde{\omega} \simeq \widetilde{g} \widetilde{\omega}$ and $\widetilde{\omega}_{1}$ from $\widetilde{x}_{0}$ to $\widetilde{x}_{1}$. Then $\widetilde{\omega}_{1}+\widetilde{\omega}$ goes from $\widetilde{x}_{0}$ to $\widetilde{x}_{2}$ and hence

$$
\Theta\left(\widetilde{x}_{2}\right)=\left\langle p\left(\widetilde{\omega}_{1}+\widetilde{\omega}\right)\right\rangle_{H}=\left\langle p \widetilde{\omega}_{1}\right\rangle_{H}+\langle p \widetilde{\omega}\rangle_{H}=\Theta\left(\widetilde{x}_{1}\right)+\langle p \widetilde{\omega}\rangle_{H} ;
$$

but $\langle p \widetilde{\omega}\rangle_{H} \in \bar{C}\left(f_{\#}, g_{\#}\right)_{x_{0}}$ since $f p \widetilde{\omega}=q \widetilde{f} \widetilde{\omega} \simeq q \widetilde{g} \widetilde{\omega}=g p \widetilde{\omega}$.

Now we assume that $P \Theta\left(\widetilde{x}_{1}\right)=P \Theta\left(\widetilde{x}_{2}\right)$. We fix paths $\widetilde{\omega}_{i}$ from $\widetilde{x}_{0}$ to $\widetilde{x}_{i}(i=1,2)$. Then $\left\langle p \widetilde{\omega}_{2}\right\rangle_{H}=\left\langle p \widetilde{\omega}_{1}\right\rangle_{H}+\langle a\rangle_{H}$ for some $\langle a\rangle \in C\left(f_{\#}, g_{\#}\right)_{x_{0}}$. Let $\widetilde{a}$ be the lift of $a$ starting at $\widetilde{x}_{1}$. Then the equality $\left\langle-p \widetilde{\omega}_{1}+p \widetilde{\omega}_{2}\right\rangle=\langle a\rangle$ implies that $-\widetilde{\omega}_{1}+\widetilde{\omega}_{2}$ and $\widetilde{a}$ have common ends, and hence $\widetilde{a}$ is a path from $\widetilde{x}_{1}$ to $\widetilde{x}_{2}$. Since a homotopy from $f a$ to $g a$ lifts to a homotopy from $\widetilde{f} \widetilde{a}$ to $\widetilde{g} \widetilde{a}, \widetilde{x}_{1}$ and $\widetilde{x}_{2}$ are Nielsen equivalent. 
(2.2) Corollary. The set $p^{-1} x_{0} \cap \Phi(\tilde{f}, \widetilde{g})$ consists of $\# C\left(f_{\#}^{H}, g_{\#}^{H}\right)_{x_{0}}$ elements. The Nielsen relation splits it into \# $\left(C\left(f_{\#}^{H}, g_{\#}^{H}\right)_{x_{0}} / \bar{C}\left(f_{\#}, g_{\#}\right)_{x_{0}}\right)$ parts each of $\# \bar{C}\left(f_{\#}, g_{\#}\right)_{x_{0}}$ elements.

Notice that the numbers involved in (2.2) do not depend on the choice of the points $x_{0} \in A, \widetilde{x}_{0} \in p^{-1} x_{0} \cap \Phi(\widetilde{f}, \widetilde{g})$ and of the lifts $\widetilde{f}, \widetilde{g}$ (such that $\left.\widetilde{x}_{0} \in \Phi(\widetilde{f}, \widetilde{g})\right)$. They only depend on the class $A$ and hence we will write $r_{A}=\# \bar{C}\left(f_{\#}, g_{\#}\right)_{x_{0}}, m_{A}=\#\left(C\left(f_{\#}^{H}, g_{\#}^{H}\right)_{x_{0}} / \bar{C}\left(f_{\#}, g_{\#}\right)_{x_{0}}\right)$.

Now suppose that $f$ and $g$ are transverse. Let $A \in \Phi^{\prime}(f, g)$ be a nondefective class ([Je2, Section 2]). Now we are going to find how many Nielsen classes of the lifts $\widetilde{f}, \widetilde{g}$ cover $A$ and we will compare their semi-indices.

Recall that for $R$-related $x, y \in \Phi(f, g), p^{-1}\{x, y\}$ splits into a sum of $R$-related pairs [DJ, (2.2)], so to simplify notation we may assume that $A=$ $\left\{x_{0}, \ldots, x_{k}\right\}$ and no two points in $A$ are $R$-related. Fix paths $\omega_{i}$ from $x_{0}$ to $x_{i}$ such that $f \omega_{i} \simeq g \omega_{i}(i=1, \ldots, k)$. Let $p^{-1} x_{0} \cap \Phi(f, g)=\left\{\widetilde{x}_{01}, \ldots, \widetilde{x}_{0 l}\right\}$.

Denote by $\widetilde{\omega}_{i j}$ the lift of $\omega_{i}$ starting at $\widetilde{x}_{0 j}$ and let $\widetilde{x}_{i j}=\widetilde{\omega}_{i j}(1)(i=$ $1, \ldots, k ; j=1, \ldots, l)$. Now $p^{-1} x_{i} \cap \Phi(\tilde{f}, \widetilde{g})=\left\{\widetilde{x}_{i 1}, \ldots, \widetilde{x}_{i l}\right\}$, the points $\left\{\widetilde{x}_{0 j}, \ldots, \widetilde{x}_{k j}\right\}$ are Nielsen equivalent and no two of them are $R$-related. On the other hand, since no $x_{i}$ is $R$-related with itself, neither is any $\widetilde{x}_{i j}$ $(j=0, \ldots, l)$.

(2.3) Remark. Under the above assumption we may write

$$
p^{-1} x_{0} \cap \Phi(\widetilde{f}, \widetilde{g})=\left\{\widetilde{x}_{01}, \ldots, \widetilde{x}_{0 r} ; \widetilde{x}_{0, r+1}, \ldots, \widetilde{x}_{0,2 r} ; \ldots ; \widetilde{x}_{0, l-r+1}, \ldots, \widetilde{x}_{0, l}\right\}
$$

where $\widetilde{x}_{0 i}$ and $\widetilde{x}_{0 j}$ lie in the same Nielsen class of $(\widetilde{f}, \widetilde{g})$ iff $(s-1) r<i, j \leq s r$ for some $s=1, \ldots, l / r$. Then the set $\widetilde{A}_{j}=\left\{\widetilde{x}_{p q}: p=0, \ldots, k ; q=r j+\right.$ $1, \ldots, r j+r\}$ is a single Nielsen class of $(\widetilde{f}, \widetilde{g})$ and $|\operatorname{ind}|\left(\widetilde{f}, \widetilde{g}: \widetilde{A}_{j}\right)=(k+1) r=$ $r \mid$ ind $\mid(f, g: A)\left(j=0, \ldots, l / r-1 ; r=r_{A}\right)$.

To formulate the final statement we denote by $\operatorname{lift}(f, g)$ the set of all lifts of the pair $(f, g)$. Then the group $\Gamma_{M} \times \Gamma_{N}$ acts on $\operatorname{lift}(f, g)$ by $(\alpha, \beta)(\tilde{f}, \widetilde{g})=$ $\beta(\tilde{f}, \widetilde{g}) \alpha^{-1}\left((\alpha, \beta) \in \Gamma_{M} \times \Gamma_{N},(\widetilde{f}, \widetilde{g}) \in \operatorname{lift}(f, g)\right)$. The orbit space will be denoted by $\operatorname{lift}^{\prime}(f, g)$. We notice that if $p \Phi(\widetilde{f}, \widetilde{g}) \cap p \Phi\left(\widetilde{f}^{\prime}, \widetilde{g}^{\prime}\right) \neq \emptyset$ then $(\widetilde{f}, \widetilde{g})$ and $\left(\widetilde{f}^{\prime}, \widetilde{g}^{\prime}\right)$ lie in the same orbit. Conversely, if they lie in the same orbit then $p \Phi(\widetilde{f}, \widetilde{g})=p \Phi\left(\widetilde{f}^{\prime}, \widetilde{g}^{\prime}\right)$. In fact, $p \Phi(\widetilde{f}, \widetilde{g})$ is a single $H^{\prime}$-Nielsen class of $(f, g)$ where $H^{\prime}=q_{\#} \pi_{1} N$ [Je1]. Thus we get a disjoint sum $\Phi(f, g)=\bigcup p \Phi(\widetilde{f}, \widetilde{g})$ where in the summation we take one representative of each orbit $[\tilde{f}, \widetilde{g}] \in$ $\operatorname{lift}^{\prime}(f, g)$. In a forthcoming paper we will show a natural bijection between $\operatorname{lift}^{\prime}(f, g)$ and the set $\nabla_{H^{\prime}}(f, g)$ defined in [Je1]. Therefore we call elements of $\operatorname{lift}^{\prime}(f, g)$ Reidemeister classes of $(f, g)$, and define $R(f, g)=\# \operatorname{lift}^{\prime}(f, g)$ to be the Reidemeister number of $(f, g)$. For the purposes of this paper it will be enough to show: 
(2.4) Lemma. If $f, g: M \rightarrow N$ and $\pi_{1} N=\mathbb{Z}_{2}$ then

$$
R(f, g)= \begin{cases}1 & \text { if } f_{\#} \neq g_{\#}, \\ 2 & \text { if } f_{\#}=g_{\#}\end{cases}
$$

Proof. The universal covering $q: \widetilde{N} \rightarrow N$ is two-fold; let $\beta: \widetilde{N} \rightarrow$ $\widetilde{N}$ denote its non-trivial transformation. We fix $(\widetilde{f}, \widetilde{g}) \in \operatorname{lift}(f, g)$. Then $\operatorname{lift}(f, g)$ consists of four elements: $(\widetilde{f}, \widetilde{g}),(\widetilde{f}, \beta \widetilde{g}),(\beta \widetilde{f}, \widetilde{g}),(\beta \widetilde{f}, \beta \widetilde{g})$. Since $(\widetilde{f}, \widetilde{g})$ and $(\beta \widetilde{f}, \beta \widetilde{g})$ lie in the same orbit and so do $(\widetilde{f}, \beta \widetilde{g})$ and $(\beta \widetilde{f}, \widetilde{g})$, it remains to show that $f_{\#}=g_{\#}$ iff $(\widetilde{f}, \widetilde{g})$ and $(\widetilde{f}, \beta \widetilde{g})$ are in distinct orbits. Fix $x \in M, \widetilde{x} \in p^{-1} x, \alpha \in \Gamma_{M}$ and a path $\widetilde{a}$ from $\widetilde{x}$ to $\alpha \widetilde{x}$. Let $a=p \widetilde{a}$. Then $(\widetilde{f} \alpha, \widetilde{g} \alpha)$ equals $(\widetilde{f}, \widetilde{g})$ or $(\beta \widetilde{f}, \beta \widetilde{g})$ iff $f_{\#} a=g_{\#} a$.

(2.5) Theorem. If $\Phi^{\prime}(f, g)$ contains no essential defective class then

$$
\sum_{(\tilde{f}, \tilde{g})} N(\tilde{f}, \widetilde{g})=\sum m_{A}
$$

where the left sum runs over representatives of orbits in $\operatorname{lift}^{\prime}(f, g)$ and the right sum over all essential classes $A \in \Phi^{\prime}(f, g)$, and $m_{A}$ is the number defined just after (2.2). In particular, if $m=m_{A}$ does not depend on the class $A$ then

$$
\sum_{(\tilde{f}, \tilde{g})} N(\widetilde{f}, \widetilde{g})=m N(f, g) .
$$

Proof. Notice that for any $A \in \Phi^{\prime}(f, g)$ there is exactly one class $[\widetilde{f}, \widetilde{g}] \in \operatorname{lift}^{\prime}(f, g)$ such that $A=p \Phi(\widetilde{f}, \widetilde{g})$. But (2.4) implies that this class is covered by $m_{A}$ classes each of semi-index $r_{A} \mid$ ind $\mid(f, g: A)$.

(2.6) Corollary. In the diagram (2.0), let $\pi_{1} N=\mathbb{Z}_{2}$, let $q$ be a universal covering and let the pair $f, g$ have no defective class. Then $m=1$ and

$$
\begin{array}{ll}
N(f, g)=N(\widetilde{f}, \widetilde{g}) & \text { if } f_{\#} \neq g_{\#}, \\
N(f, g)=N(\widetilde{f}, \widetilde{g})+N(\widetilde{f}, \beta \widetilde{g}) & \text { if } f_{\#}=g_{\#},
\end{array}
$$

where $\beta$ denotes the unique non-trivial transformation of the two-fold covering $q$.

3. The general method. In this section we give a general method to compute the Nielsen number of a pair $f, g: M \rightarrow \mathbb{R} P^{n}$ where $M$ denotes an $n$-manifold.

First recall that the map $q: S^{n} \rightarrow \mathbb{R} P^{n}$ identifying antipodal points is the universal covering map and

$$
\pi_{i} \mathbb{R} P^{n}= \begin{cases}\mathbb{Z}_{2} & \text { for } i=1, \\ \mathbb{Z} & \text { for } i=n, \\ 0 & \text { for } i=2, \ldots, n-1\end{cases}
$$


One can also prove

(3.1) LEMmA. The action of the non-zero element of $\pi_{1} \mathbb{R} P^{n}$ on $\pi_{n} \mathbb{R} P^{n}$ is multiplication by $(-1)^{n+1}$.

Let $f, g: M \rightarrow N$ be a pair of maps between smooth $n$-dimensional manifolds. Denote by $\pi_{1}^{+} M\left(\pi_{1}^{+} N\right)$ the subgroup of orientation-preserving elements of $\pi_{1} M\left(\pi_{1} N\right)$. For $x \in \Phi(f, g)$ we put $C^{+}\left(f_{\#}, g_{\#}\right)_{x}=C\left(f_{\#}, g_{\#}\right)_{x} \cap$ $\pi_{1}^{+} M$. Recall [Je2, (2.6)] that the Nielsen class containing $x$ is not defective iff

$$
C^{+}\left(f_{\#}, g_{\#}\right)_{x}=C\left(f_{\#}, g_{\#}\right)_{x} \cap f_{\#}^{-1}\left(\pi_{1}^{+} N\right) .
$$

(3.3) LEMmA. If $\pi_{1} N$ is abelian then either all Nielsen classes are defective or none is.

Proof. Let $x_{0}, x_{1} \in \Phi(f, g)$ and let $r$ be a path from $x_{0}$ to $x_{1}$. Then the restrictions of $h_{\langle r\rangle}: \pi_{1}\left(M, x_{0}\right) \rightarrow \pi_{1}\left(M, x_{1}\right), h_{\langle r\rangle}\langle a\rangle=\langle-r+a+r\rangle$ :

$$
\begin{aligned}
& C^{+}\left(f_{\#}, g_{\#}\right)_{x_{0}}=C\left(f_{\#}, g_{\#}\right)_{x_{0}} \cap f_{\#}^{-1} \pi_{1}^{+}\left(N, f x_{0}\right) \\
& h_{\langle r\rangle} \downarrow \quad \downarrow h_{\langle r\rangle} \quad h_{\langle r\rangle} \downarrow \\
& C^{+}\left(f_{\#}, g_{\#}\right)_{x_{1}}=C\left(f_{\#}, g_{\#}\right)_{x_{1}} \cap f_{\#}^{-1} \pi_{1}^{+}\left(N, f x_{1}\right)
\end{aligned}
$$

are isomorphisms since $\pi_{1} N$ is abelian.

Now we consider again the maps into $\mathbb{R} P^{n}$. We will consider three cases: (3.4), (3.5), (3.9):

(3.4) The equality (3.2) holds for an $x \in \Phi(f, g)$.

Then by (3.3) there is no defective class. We find a finite covering $p$ : $\widetilde{M} \rightarrow M$ admitting lifts

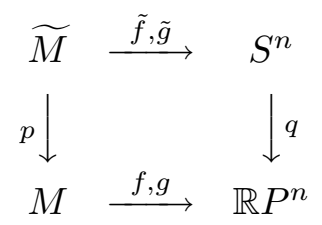

and we apply (2.6). Such a covering always exists since $\pi_{1} M$ is finitely generated: we denote by $H$ the subgroup of elements divisible by 2 and take the covering corresponding to $H$.

(3.5) The equality (3.2) does not hold and $f_{\#} \neq g_{\#}$.

Then there is at most one Nielsen class and this class is defective. Thus $N(f, g)=L_{2}(f, g)$.

To discuss the last case we will need the following classification of maps into real projective spaces (see [O, Section 27]). 
(3.6) Lemma. The homotopy classes of maps $f:(M, x) \rightarrow\left(\mathbb{R} P^{n}, y\right)$ are in one-to-one correspondence with the set of pairs $(\Theta, h)$ where $\Theta$ : $\pi_{1}(M, x) \rightarrow \pi_{1}\left(\mathbb{R} P^{n}, y\right)$ is a homomorphism and $h \in H^{n}\left(M, \Theta^{*} \pi_{n}\right)$ where $\Theta^{*} \pi_{n}$ denotes the local system induced from $\pi_{n} \mathbb{R} P^{n}$ by $\Theta$. Moreover,

$$
H^{n}\left(M, \Theta^{*} \pi_{n}\right)= \begin{cases}\mathbb{Z} & \text { if } \Theta^{-1} \pi_{1}^{+} \mathbb{R} P^{n}=\pi_{1}^{+} M \\ \mathbb{Z}_{2} & \text { otherwise. }\end{cases}
$$

Consider a pair of maps $f, f^{\prime}:\left(M, x_{0}\right) \rightarrow\left(\mathbb{R} P^{n}, y_{0}\right)$ satisfying $f_{\#}=f_{\#}^{\prime}$. Then $f$ and $f^{\prime}$ are homotopic on the 1-skeleton. Since $\pi_{i} \mathbb{R} P^{n}=0$ for $i=2, \ldots, n-1$, there is no obstruction to a homotopy between these maps up to the $(n-1)$-skeleton. Thus we may assume that $f$ and $f^{\prime}$ are equal on $M-\sigma$ where $\sigma \subset M$ is an $n$-cell. Now $f^{\prime}=f \# s$ for a map $s: S^{n} \rightarrow \mathbb{R} P^{n}$ (here \# denotes the connected sum $f \# s: M \# S^{n}=M \rightarrow \mathbb{R} P^{n}$ ).

(3.7) Lemma. Let $f, g: M \rightarrow N$ and $s: S^{n} \rightarrow N$. Then

$$
\begin{aligned}
L(f, g \# s) & =L(f, g)+\operatorname{deg} s & & \text { for } M, N \text { oriented, } \\
L_{2}(f, g \# s) & =L_{2}(f, g)+\operatorname{deg}_{2} s & & \text { without this assumption. }
\end{aligned}
$$

Proof. Since the Lefschetz number equals the coincidence index it is enough to see how the last varies. We may assume that for a closed $n$-ball $K \subset M$ we have $f(K)=y_{0}, g(K)=y_{1} \neq y_{0}$ and that $(g \# s)(x)=g(x)$ for $x \notin K$. Now $x \in K$ is a coincidence point iff $s(x)=y_{0}$. But any $y \in N$ is covered $\operatorname{deg} s$ times by the map $s$ (algebraically counting). Thus the indices of $(f, g \# s)$ and $(f, g)$ differ by $\operatorname{deg} s$.

(3.8) LEMMA. If $f_{\#}=g_{\#}$ then $x, x^{\prime} \in \Phi(f, g)$ are Nielsen equivalent iff $f \omega \simeq g \omega$ for any path $\omega$ from $x$ to $x^{\prime}$.

Proof. Let $f \omega \simeq g \omega$ and assume that $\omega^{\prime}$ is another path from $x$ to $x^{\prime}$. Then $\omega^{\prime} \simeq \alpha+\omega$ for some loop $\alpha$ based at $x$. Thus $f \omega^{\prime} \simeq f \alpha+f \omega \simeq$ $g \alpha+g \omega \simeq g \omega^{\prime}$.

Consider again $f, g: M \rightarrow \mathbb{R} P^{n}$ which do not satisfy (3.2) and $f_{\#}=g_{\#}$. If $f \simeq g$ then there is only one Nielsen class and it is defective. Thus $N(f, g)=L_{2}(f, g)$ in this case.

Finally, suppose (3.2) does not hold, $f_{\#}=g_{\#}$ but $f \neq g$. We may assume (after a local homotopy) that $f$ sends a ball $K$ into a point $x_{0}$. Let $f^{\prime}$ be a map homotopic to $f$ such that $f^{\prime}(K)=x_{1}, x_{0} \neq x_{1}$. Let $q: S^{n} \rightarrow \mathbb{R} P^{n}$ denote the universal covering. By (3.6) we may assume that $g=f^{\prime} \# q$. Since $\Phi\left(f, f^{\prime}\right)$ is one Nielsen class, all points in $\Phi(f, g)-K$ are Nielsen equivalent. Moreover, there are two other coincidence points in $K$. These are not Nielsen equivalent since they correspond to antipodal points in $q^{-1}\left(x_{0}\right)$ (Lemma (3.8)). Now one of them is an essential Nielsen class while the remaining coincidence points form another class of semi-index $L_{2}(f, g)+1 \in \mathbb{Z}_{2}$. Thus we obtain 
(3.9) Corollary. If $f_{\#}=g_{\#}$ and (3.2) does not hold then

$$
N(f, g)= \begin{cases}L_{2}(f, g) & \text { for } f \simeq g, \\ 2 & \text { for } f \not z, L_{2}(f, g)=0, \\ 1 & \text { for } f \not g, L_{2}(f, g)=1 .\end{cases}
$$

It follows from (3.4), (3.5) and (3.9) that to compute $N(f, g)$ it is enough to know the Nielsen number of maps into $S^{n}$ and the $\mathbb{Z}_{2}$-Lefschetz number. Now we will solve the first problem and the latter will be computed for some special manifolds in the next sections.

Consider $f, g: M \rightarrow S^{n}(n>1)$. Since $S^{n}$ is simply connected, $N(f, g) \leq 1$.

(3.10) LEMMA. If $M$ is an orientable closed $n$-manifold then

$$
N(f, g)= \begin{cases}0 & \text { if } f \text { is homotopic to } \alpha g, \\ 1 & \text { otherwise, }\end{cases}
$$

where $\alpha$ denotes the antipodal map.

Proof. Since ind $(f, g)=L(f, g)=\operatorname{deg} g+(-1)^{n} \operatorname{deg} f$, ind $(f, g)=0$ iff $\operatorname{deg} g=(-1)^{n+1} \operatorname{deg} f$ but the last holds exactly for $f$ homotopic to $\alpha g$.

(3.11) LEMMA. If in the above lemma $M$ is not orientable then

$$
N(f, g)= \begin{cases}1 & \text { if } f \text { and } g \text { are not homotopic, } \\ 0 & \text { otherwise. }\end{cases}
$$

Proof. By the Hopf theorem there are exactly two homotopy classes of maps from a non-orientable manifold $M$ into $S^{n}$. To obtain the non-trivial map we fix an $n$-cell $\sigma \subset M$ and we send $M-\sigma$ into a point $x_{0}$ and the interior of $\sigma$ diffeomorphically onto $S^{n}-x_{0}$. Now let $f$ be non-trivial, of the above form, and let $g$ be constant, $g(M)=x_{1} \neq x_{0}$. Then $f$ and $g$ are transverse and $\Phi(f, g)$ consists of one point, therefore $\mid$ ind $\mid(f, g)=1$ implies $N(f, g)=1$.

If both $f$ and $g$ are constant then obviously $N(f, g)=0$.

Now let $f=g$ be non-trivial. Assume first that $n$ is odd. Then the identity map is homotopic to a fixed point free map $\alpha$. Thus $g=f \simeq \alpha f$ and $\Phi(f, \alpha f)=\emptyset$ implies $N(f, g)=N(f, \alpha f)=0$. Let now $n$ be even. The only Nielsen class of $f=g$ is defective since any orientation-reversing loop on $M$ is sent to a null homotopic one. We may assume that $x_{0} \notin$ $\{(0, \ldots, 0,+1),(0, \ldots, 0,-1)\}$ and we notice that the map $h: S^{n} \rightarrow S^{n}$, $h\left(x_{1}, \ldots, x_{n+1}\right)=\left(-x_{1}, \ldots,-x_{n}, x_{n+1}\right)$, is homotopic to the identity since $\operatorname{deg} h=(-1)^{n}=1$. Now $g=f \simeq h f$, the last two maps are transverse and $\Phi(f, h f)=\left\{f^{-1}(0, \ldots, 0,+1), f^{-1}(0, \ldots, 0,-1)\right\}$ consists of exactly two points. Since this class is defective, $\mid$ ind $\mid(f, g)=0$ and $N(f, g)=0$. 
Finally, we will apply the above methods to compute the Nielsen number of $f, g: S^{n} \rightarrow \mathbb{R} P^{n}$. Since $S^{n}$ is simply connected, no class in $\Phi^{\prime}(f, g)$ is defective and we may apply (3.4). We fix lifts

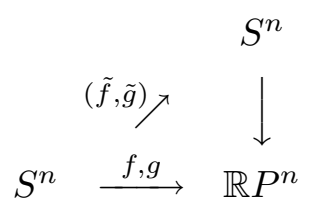

and we notice that $\pi_{1} S^{n}=0$ implies $m=1$ (in (2.5)) and $R(f, g)=2$, hence $N(f, g)=N(\widetilde{f}, \widetilde{g})+N(-\widetilde{f}, \widetilde{g})$.

(3.12) Corollary. Let $f, g: S^{n} \rightarrow \mathbb{R} P^{n}$. Then

$$
N(f, g)= \begin{cases}0 & \text { for }(f \simeq g \simeq \text { const, } n \text { even }) \text { or }(f \simeq g, n \text { odd }), \\ 1 & \text { for } f \simeq g \neq \text { const, } n \text { even, } \\ 2 & \text { for } f \not z g .\end{cases}
$$

Proof. Let $n$ be odd. Then $N(f, g)=N(\tilde{f}, \widetilde{g})+N(\widetilde{f},-\widetilde{g})=2 N(\tilde{f}, \widetilde{g})$. Thus

$$
N(f, g)= \begin{cases}0 & \text { if } f \simeq g \\ 2 & \text { otherwise }\end{cases}
$$

since $\tilde{f} \simeq \widetilde{g}$ iff $f \simeq g$.

Let now $n$ be even. Then $N(f, g)=N(\widetilde{f}, \widetilde{g})+N(\widetilde{f},-\widetilde{g})$ and

$$
N(f, g)= \begin{cases}0 & \text { if } f \simeq g \simeq \text { const }, \\ 1 & \text { if } f \simeq g \neq \text { const } \\ 2 & \text { if } f \not z g,\end{cases}
$$

since $f \simeq g$ iff $\tilde{f} \simeq \mp \widetilde{g}$ and $N(\widetilde{f}, \widetilde{g})=0$ iff $\tilde{f} \simeq-\widetilde{g}$.

4. The coincidence Nielsen number for maps from an evendimensional torus. In this and the next sections we assume that $n$ is even; the odd case is easier and will be discussed in Section 6 .

Now we apply the results of the last section to maps from the $n$-torus $T^{n}=\left(S^{1}\right)^{n}=([0,1] /\{0=1\})^{n}$ into $\mathbb{R} P^{n}$. We fix the points $p_{0}=\{0=$ $1\} \in S^{1}, x_{0}=\left(p_{0}, \ldots, p_{0}\right) \in\left(S^{1}\right)^{n}=T^{n}$ and $y_{0}=[1,0, \ldots, 0] \in \mathbb{R} P^{n}$. Let $a_{j}(t)$ denote the canonical generators of $\pi_{1}\left(T^{n}, x_{0}\right)$ given by $a_{j}(t)=$ $\left(p_{0}, \ldots, p_{0}, \exp (2 \pi t i), p_{0}, \ldots, p_{0}\right) \in T^{n}(j=1, \ldots, n)$. Let $\Theta: \pi_{1}\left(T^{n}, x_{0}\right) \rightarrow$ $\pi_{1}\left(\mathbb{R} P^{n}, y_{0}\right)=\mathbb{Z}_{2}$ be a homomorphism and let $\Lambda_{\Theta}=\{j \in\{1, \ldots, n\}$ : $\left.\Theta\left(a_{j}\right) \neq 0\right\}$. Then the formula

$$
f_{\Theta}\left(t_{1}, \ldots, t_{n}\right)=\left[\exp \left(\sum_{k \in \Lambda_{\Theta}} t_{k} \pi i\right), 0, \ldots, 0\right]
$$


gives a map $f_{\Theta}: T^{n} \rightarrow \mathbb{R} P^{n}$ such that $f_{\Theta \#}=\Theta$. Now fix a map $f:\left(T^{n}, x_{0}\right) \rightarrow\left(\mathbb{R} P^{n}, y_{0}\right)$ and let $\Theta=f_{\#}$. Since $f_{\#}=f_{\Theta \#}$, we may assume by (3.6) that $f=f_{\Theta} \# s$ for a map $s: S^{n} \rightarrow \mathbb{R} P^{n}$. Thus $f$ sends the $(n-1)$-skeleton into the 1-skeleton and all induced homology and cohomology homomorphisms are zero in dimensions $k=2, \ldots, n-1$. On the other hand, we notice that $\operatorname{deg}_{2} f_{\Theta}=0$ since the whole image of $f_{\Theta}$ lies in the 1-skeleton. Moreover, $\operatorname{deg}_{2} s=0$ since $s$ admits a lift

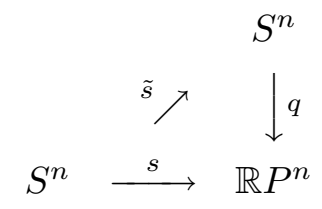

and $\operatorname{deg}_{2} s=\operatorname{deg}_{2} q \widetilde{s}=\operatorname{deg}_{2} q \operatorname{deg}_{2} \widetilde{s}$ but $\operatorname{deg}_{2} q=0$ since $q$ is two-fold. Thus $\operatorname{deg}_{2} f=\operatorname{deg}_{2}\left(f_{\Theta} \# s\right)=\operatorname{deg}_{2} f_{\Theta}+\operatorname{deg}_{2} s=0$ and $f_{*}: H_{n}\left(T^{n}, \mathbb{Z}_{2}\right) \rightarrow$ $H_{n}\left(\mathbb{R} P^{n}, \mathbb{Z}_{2}\right), f^{*}: H^{n}\left(\mathbb{R} P^{n}, \mathbb{Z}_{2}\right) \rightarrow H^{n}\left(T^{n}, \mathbb{Z}_{2}\right)$ are also zero homomorphisms.

Now we are in a position to compute the $\mathbb{Z}_{2}$-Lefschetz number of $f, g$ : $T^{n} \rightarrow \mathbb{R} P^{n}$. We consider the squares

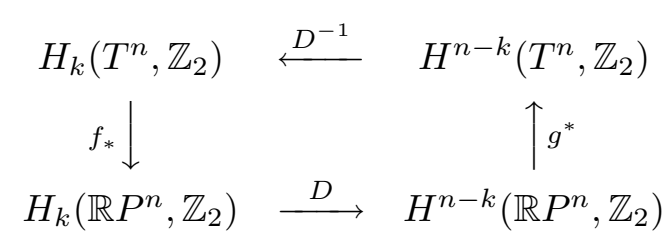

If $n>2$ then for any $k$ either $1<k$ or $1<n-k$ so in any case at least one of the vertical homomorphisms is zero and $L_{2}(f, g)=0$.

If $n=2$ then the above diagram may give a non-zero homomorphism for $k=1$. In this case we write $f_{*} a_{i}=k_{i} a, g_{*} a_{i}=k_{i}^{\prime} a$, hence $f^{*} \bar{a}=k_{1} \bar{a}_{1}+k_{2} \bar{a}_{2}$, $g^{*} \bar{a}=k_{1}^{\prime} \bar{a}_{1}+k_{2}^{\prime} \bar{a}_{2}\left(\right.$ here $a_{1}, a_{2} \in H_{1}\left(T^{n}, \mathbb{Z}_{2}\right), \bar{a}_{1}, \bar{a}_{2} \in H^{1}\left(T^{n}, \mathbb{Z}_{2}\right), a \in$ $H_{1}\left(\mathbb{R} P^{n}, \mathbb{Z}_{2}\right), \bar{a} \in H^{1}\left(\mathbb{R} P^{n}, \mathbb{Z}_{2}\right)$ denote the canonical generators $)$. Thus

$$
L_{2}(f, g)=\operatorname{tr}\left(D^{-1} g^{*} D f_{*}\right)=\operatorname{det}\left[\begin{array}{ll}
k_{1} & k_{2} \\
k_{1}^{\prime} & k_{2}^{\prime}
\end{array}\right] \in \mathbb{Z}_{2} .
$$

Now we may compute the Nielsen number; we consider three cases according to Section 3.

(a) The equality (3.2) holds.

(a1) Let $f_{\#} \neq g_{\#}$. Then one of the homomorphisms $f_{\#}, g_{\#}$ must be zero. Indeed, suppose otherwise. Then $f_{\#} a_{i} \neq 0, g_{\#} a_{j} \neq 0$ for some $i, j=$ $1, \ldots, n$. If moreover $g_{\#} a_{i} \neq 0$ then $a_{i} \in C^{+}\left(f_{\#}, g_{\#}\right)$ but $f_{\#} a_{i}=g_{\#} a_{i}=a$ is orientation-reversing on $\mathbb{R} P^{n}$, contradicting (3.2). If $f_{\#} a_{j}=g_{\#} a_{i}=0$ then $a_{i}+a_{j}$ contradicts $(3.2)$. 
Let $p: T^{n} \rightarrow T^{n}$ be a $2^{n}$-fold covering corresponding to the subgroup of elements of $\pi_{1} T^{n}$ divisible by 2 . Then any pair of maps $f, g$ admits lifts

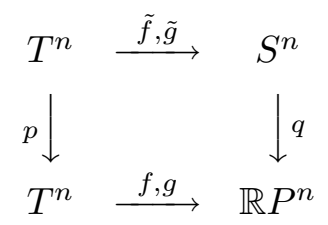

and (2.6) implies $N(f, g)=N(\tilde{f}, \widetilde{g})$.

We may assume that $f_{\#}=0$ and $g_{\#} b \neq 0$ for some $b \in \pi_{1} T^{n}$ not divisible by 2 . Let $\beta$ be the transformation of the covering $p: T^{n} \rightarrow T^{n}$ determined by $b$. Then the diagram

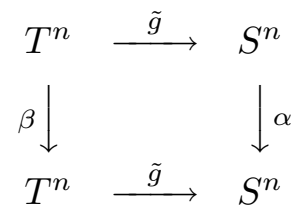

commutes ( $\alpha$ denotes the antipodism). Now, by (1.2), $\operatorname{deg} \widetilde{g}=0$, so $(3.10)$ implies

$$
N(\widetilde{f}, \widetilde{g})= \begin{cases}0 & \text { if } \operatorname{deg} \widetilde{f}=0, \\ 1 & \text { if } \operatorname{deg} \widetilde{f} \neq 0 .\end{cases}
$$

On the other hand, since $f_{\#}=0$, there exists a map $\tilde{f}^{\prime}$ such that the diagram

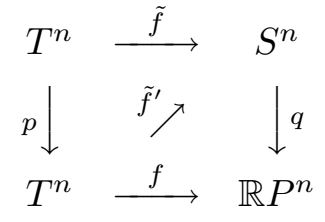

commutes. Since $\operatorname{deg} \widetilde{f}=\operatorname{deg}\left({\widetilde{f^{\prime}}}^{\prime} p\right)=\operatorname{deg} \widetilde{f^{\prime}} \operatorname{deg} p=2^{n} \operatorname{deg} \widetilde{f}^{\prime}, N(\widetilde{f}, \widetilde{g})=0$ iff $\operatorname{deg} \widetilde{f^{\prime}}=0$ but then $\widetilde{f}^{\prime}$ is null homotopic, and hence so is $f=q \widetilde{f}^{\prime}$. Thus we get

$$
N(f, g)= \begin{cases}0 & \text { if one of the maps } f, g \text { is null homotopic, } \\ 1 & \text { otherwise. }\end{cases}
$$
lifts

(a2) If $f_{\#}=g_{\#}$ then (3.2) means $f_{\#}=g_{\#}=0$ and the maps $f, g$ admit

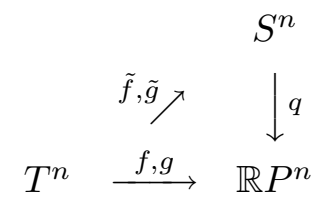


Then by (2.6), $N(f, g)=N(\widetilde{f}, \widetilde{g})+N(\widetilde{f},-\widetilde{g})$ and hence

$$
N(f, g)= \begin{cases}0 & \text { if } f \simeq g \simeq \text { const }, \\ 1 & \text { if } f \simeq g \neq \text { const }, \\ 2 & \text { if } f \not z g .\end{cases}
$$

(b) (3.2) does not hold and $f_{\#} \neq g_{\#}$. Then there is only one Reidemeister class and it is defective. If $n \geq 3$ then $L_{2}(f, g)=0$ and [Je2, (2.3)] implies $N(f, g)=0$. If $n=2$ then one can check that our assumptions are fulfilled exactly for

$$
\left[\begin{array}{ll}
k_{1} & k_{2} \\
k_{1}^{\prime} & k_{2}^{\prime}
\end{array}\right]=\left[\begin{array}{ll}
0 & 1 \\
1 & 1
\end{array}\right],\left[\begin{array}{ll}
1 & 0 \\
1 & 1
\end{array}\right],\left[\begin{array}{ll}
1 & 0 \\
0 & 1
\end{array}\right],\left[\begin{array}{ll}
0 & 1 \\
1 & 0
\end{array}\right],\left[\begin{array}{ll}
1 & 1 \\
0 & 1
\end{array}\right],\left[\begin{array}{ll}
1 & 1 \\
1 & 0
\end{array}\right] .
$$

But now in any case

$$
L_{2}(f, g)=\operatorname{det}\left[\begin{array}{ll}
k_{1} & k_{2} \\
k_{1}^{\prime} & k_{2}^{\prime}
\end{array}\right] \neq 0 \in \mathbb{Z}_{2}
$$

and hence $N(f, g)=1$.

(c) (3.2) does not hold and $f_{\#}=g_{\#}$. Then $L_{2}(f, g)=0$ and (3.9) imply

$$
N(f, g)= \begin{cases}0 & \text { if } f \simeq g \\ 2 & \text { otherwise. }\end{cases}
$$

5. The coincidence Nielsen number for maps from an evendimensional projective space. Let $n \in \mathbb{N}$ be even. Recall that

$$
H_{k}\left(\mathbb{R} P^{n}, \mathbb{Z}_{2}\right)=H^{k}\left(\mathbb{R} P^{n}, \mathbb{Z}_{2}\right)= \begin{cases}\mathbb{Z}_{2} & \text { for } k \leq n, \\ 0 & \text { for } k>n,\end{cases}
$$

and $H^{*}\left(\mathbb{R} P^{n}, \mathbb{Z}_{2}\right)=\mathbb{Z}_{2}[a] /\left(a^{n+1}\right)$ as algebras. We denote by $a_{k} \in H_{k}\left(\mathbb{R} P^{n}\right.$, $\left.\mathbb{Z}_{2}\right), a^{k} \in H^{k}\left(\mathbb{R} P^{n}, \mathbb{Z}_{2}\right)$ the non-trivial elements, $k=0, \ldots, n$. Consider a map $f: \mathbb{R} P^{n} \rightarrow \mathbb{R} P^{n}$; let $f_{*} a_{1}=c a_{1}\left(c \in \mathbb{Z}_{2}\right)$. Then $f^{*} a^{1}=c a^{1}$, hence $f^{*} a^{k}=c a^{k}$, which implies $f_{*} a_{k}=c a_{k}$ (universal coefficients formulae), $k=1, \ldots, n$.

Consider $f, g: \mathbb{R} P^{n} \rightarrow \mathbb{R} P^{n}$. We now determine the $\mathbb{Z}_{2}$-Lefschetz number of this pair. Let $f_{*} a_{1}=c a_{1}, g_{*} a_{1}=c^{\prime} a_{1}$. Then the above discussion implies that in the diagrams

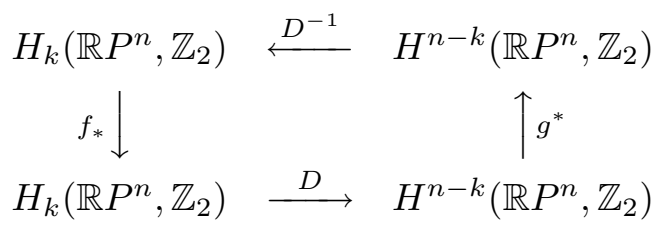

we have

$$
D^{-1} g^{*} D f_{*}\left(a_{k}\right)= \begin{cases}c^{\prime} a_{0} & \text { for } k=0, \\ c c^{\prime} a_{k} & \text { for } k=1, \ldots, n-1, \\ c a_{n} & \text { for } k=n\end{cases}
$$


Thus $L_{2}(f, g)=c^{\prime}+(n-1) c c^{\prime}+c=c^{\prime}+c c^{\prime}+c \in \mathbb{Z}_{2}$ (since $n$ is even) and finally

$$
L_{2}(f, g)= \begin{cases}0 & \text { if } c=c^{\prime}=0 \\ 1 & \text { otherwise }\end{cases}
$$

Now we compute the Nielsen number. First we assume that $f_{\#} \neq g_{\#}$. Then there is only one Reidemeister class and $L_{2}(f, g)=1$ implies $N(f, g)=$ 1. Let now $f_{\#}=g_{\#}=0$. If moreover $f$ and $g$ are homotopic then there is only one Nielsen class and it is defective, so $L_{2}(f, g)=0$ implies $N(f, g)=$ 0 . If $f$ and $g$ are not homotopic then $N(f, g)=2$ by (3.9). Finally, let $f_{\#}=g_{\#}=$ id. Then no class is defective and we may use lifts:

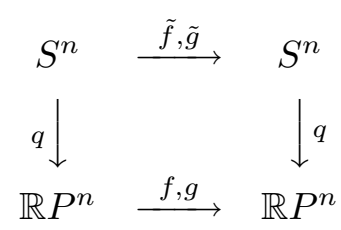

Now $\widetilde{f}, \widetilde{g}$ are odd maps $(\widetilde{f}(-\widetilde{x})=-\widetilde{f}(\widetilde{x}))$ and hence their degrees are odd numbers. On the other hand, the number $m$ from (2.5) equals one and now Lemma (3.1) implies

$$
\begin{aligned}
N(f, g)=N(\widetilde{f}, \widetilde{g})+N(\widetilde{f},-\widetilde{g}) & = \begin{cases}1 & \text { if }|\operatorname{deg} \widetilde{f}|=|\operatorname{deg} \widetilde{g}|, \\
2 & \text { otherwise }\end{cases} \\
& = \begin{cases}1 & \text { if } f \simeq g, \\
2 & \text { otherwise. }\end{cases}
\end{aligned}
$$

Finally, we get

(5.1) CoRollary. For any pair of maps $f, g: \mathbb{R} P^{n} \rightarrow \mathbb{R} P^{n}$ (with $n$ even)

$$
N(f, g)= \begin{cases}0 & \text { if } f_{\#}=g_{\#}=0 \text { and } f \simeq g, \\ 1 & \text { if } f_{\#} \neq g_{\#} \text { or }\left(f_{\#}=g_{\#}=\text { id and } f \simeq g\right), \\ 2 & \text { if } f_{\#}=g_{\#} \text { and } f \neq g .\end{cases}
$$

In particular, for $g=\mathrm{id}$ we obtain a formula for the fixed point Nielsen number of a self-map $f$ of $\mathbb{R} P^{n}$ ( $n$ even):

$$
N(f)= \begin{cases}2 & \text { if } f_{\#}=\mathrm{id} \text { and } f \neq \mathrm{id}, \\ 1 & \text { otherwise. }\end{cases}
$$

6. The coincidence Nielsen number for maps from an odddimensional manifold. The computation of the two last sections could be modified to cover also the odd-dimensional case. But this turns out to be 
easier since then $\mathbb{R} P^{n}$ is orientable. Let $n=2 k+1$. The cyclic homotopy $H: \mathbb{R} P^{n} \times I \rightarrow \mathbb{R} P^{n}$,

$$
H\left(\left[z_{1}, \ldots, z_{k+1}\right], t\right)=\left[z_{1} \exp (\pi t i), \ldots, z_{k+1} \exp (\pi t i)\right],
$$

shows that $\mathbb{R} P^{n}$ is a Jiang space [J]. Let $f, g: M \rightarrow \mathbb{R} P^{n}$ be maps from an $n$-dimensional orientable manifold. Then Lemma (6.6) of [Je1] implies that

$$
N(f, g)= \begin{cases}0 & \text { if } L(f, g)=0, \\ R(f, g) & \text { if } L(f, g) \neq 0,\end{cases}
$$

so it remains to compute the ordinary Lefschetz number. Since

$$
H_{k}\left(\mathbb{R} P^{n}, \mathbb{Q}\right)=H^{k}\left(\mathbb{R} P^{n}, \mathbb{Q}\right)= \begin{cases}\mathbb{Q} & \text { if } k=0, n \\ 0 & \text { otherwise }\end{cases}
$$

in the diagrams

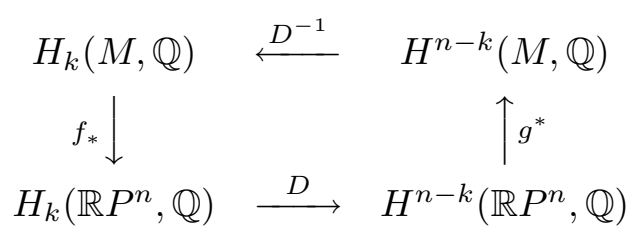

we have $\Theta_{0}=\operatorname{deg} g, \Theta_{n}=\operatorname{deg} f$ and $\Theta_{k}=0$ for other $k$, which implies $L(f, g)=\operatorname{deg} g-\operatorname{deg} f$.

(6.1) CoRollary. Let $M$ be an oriented $n$-manifold (with $n$ odd) and let $f, g: M \rightarrow \mathbb{R} P^{n}$. Then

$$
N(f, g)= \begin{cases}0 & \text { if } \operatorname{deg} f=\operatorname{deg} g, \\ 1 & \text { if } \operatorname{deg} f \neq \operatorname{deg} g, f_{\#} \neq g_{\#}, \\ 2 & \text { if } \operatorname{deg} f \neq \operatorname{deg} g, f_{\#}=g_{\#} .\end{cases}
$$

Proof. Check case by case. Combine (2.4) with the arguments of this section.

\section{References}

[DJ] R. Dobreńko and J. Jezierski, The coincidence Nielsen number on non-orientable manifolds, Rocky Mountain J. Math., to appear.

[Je1] J. Jezierski, The Nielsen number product formula for coincidences, Fund. Math. 134 (1989), 183-212.

[Je2] - The semi-index product formula, this issue, 99-120.

[J] B. J. Jiang, Lectures on the Nielsen Fixed Point Theory, Contemp. Math. 14, Amer. Math. Soc., Providence, R.I., 1983.

[M] Ch. Maxwell, Coincidences of maps, in: Global Analysis-Analysis on Manifolds, Teubner Texte zur Math. 57, Teubner, Leipzig 1983, 216-237.

[O] P. Olum, Obstructions to extensions and homotopies, Ann. of Math. 52 (1950), $1-50$. 
[V] J. Vick, Homology Theory, Academic Press, New York 1973.

DEPARTMENT OF MATHEMATICS

UNIVERSITY OF AGRICULTURE

NOWOURSYNOWSKA 166

02-766 WARSZAWA, POLAND

Received 23 July 1990;

in revised form 8 April 1991 